\title{
THE MIXED BIVARIATE HOFMANN DISTRIBUTION
}

\author{
BY \\ J.F. WALHIN *• AND J. PARIS* \\ * Université Catholique de Louvain \\ - Secura Belgian Re
}

\begin{abstract}
In this paper we study a class of Mixed Bivariate Poisson Distributions by extending the Hofmann Distribution from the univariate case to the bivariate case.

We show how to evaluate the bivariate aggregate claims distribution and we fit some insurance portfolios given in the literature.

This study typically extends the use of the Bivariate Independent Poisson Distribution, the Mixed Bivariate Negative Binomial and the Mixed Bivariate Poisson Inverse Gaussian Distribution.
\end{abstract}

\section{KEYWORDS}

Mixed Bivariate Independent Poisson Distributions, Hofmann Distribution, maximum likelihood, aggregate claims distribution, recursive algorithm, stable algorithm.

\section{INTRODUCTION}

In this paper we study a family of bivariate counting distributions. These distributions are of interest in actuarial sciences when one wants to work with frequencies of dependent variables such as material damage and bodily injury claims in third party liability automobile insurance.

The general family of bivariate distributions we present in this paper has the following particular cases: the Mixed Bivariate Negative Binomial Distribution (MBNBD) and the Mixed Bivariate Poisson Inverse Gaussian Distribution (MBPIGD). These particular cases have already been discussed in Besson and Partrat (1992) and in Partrat (1994).

By extending the univariate Hofmann Distribution described in Walhin and Paris (2000b), we give a general setting for studying Mixed Bivariate Independent Poisson Distributions.

Note that we use the term Mixed Bivariate Independent Poisson Distribution in order to stress on the fact that it is a Bivariate Distribution obtained by mixing the Bivariate Independent Poisson Distribution. 
The bivariate version of the Hofmann Distribution obtained by mixing the Bivariate Independent Poisson Distribution will be called the Mixed Bivariate Hofmann Distribution. It remains important to stress on the "Mixed" because Bivariate Hofmann Distributions can also be constructed via the trivariate reduction method (see Walhin and Paris (2000c)).

The rest of the paper is organized as follows. Section 2 reviews the concept of bivariate ordinary generating functions. Section 3 describes the Mixed Bivariate Independent Poisson Distribution. Section 4 extends the univariate Hofmann Distribution to the bivariate case. Section 5 addresses the problem of estimating the parameters of the distribution. Section 6 gives a stable recursion for the aggregate claims distribution which is based on a two-stage algorithm. Section 7 gives the fits for the data sets given in Besson and Partrat (1992) and in Partrat (1994). The two-stage algorithm is also applied. Section 8 gives the conclusion.

\section{BIVARIATE ORDINARY GENERATING FUNCTIONS}

In the following sections we will use the concept of bivariate ordinary generating functions. This is a generalization of the ordinary generating functions (see Panjer and Willmot (1992) for an application in actuarial sciences).

Let us assume a sequence $\left\{a_{n, m}, n \in \mathbb{Z}, m \in \mathbb{Z}\right\}$ of real numbers.

The ordinary generating function of this sequence is defined as

$$
T_{a_{n, m}}(u, v)=\sum_{n=0}^{\infty} \sum_{m=0}^{\infty} a_{n, m} u^{n} v^{m}
$$

Obviously $u$ and $v$ must be chosen in such a way that the sum exists.

Ordinary generating functions have the following nice properties:

- There is a one-to-one correspondence between $\left\{a_{n, m}, n \in \mathbb{Z}, m \in \mathbb{Z}\right\}$ and

$$
\begin{aligned}
& T_{a_{n, m}}(u, v) . \\
& -a_{n, m}=\left.\frac{1}{n ! m !} \frac{\partial^{n} \partial^{m} T_{a_{n, m}}(u, v)}{\partial u^{n} \partial v^{m}}\right|_{u=0, v=0}
\end{aligned}
$$$$
-c_{n, m}=\alpha a_{n, m}+\beta b_{n, m} \Leftrightarrow T_{c_{n, m}}(u, v)=\alpha T_{a_{n, m}}(u, v)+\beta T_{b_{n, m}}(u, v) \text {. }
$$$$
\text { - } c_{n, m}=\sum_{k=0}^{n} \sum_{l=0}^{m} a_{k, l} b_{n-k, m-l} \Leftrightarrow T_{c_{n, m}}(u, v)=T_{a_{n, m}}(u, v) T_{b_{n, m}}(u, v) \text {. }
$$

- $T_{n a_{n, m}}(u, v)=u \frac{\partial}{\partial u} T_{a_{n, m}}(u, v)$.

The philosophy behind using ordinary generating functions is the following:

- look for a relation between some sequences $a_{n, m}, b_{n, m}, c_{n, m}, \ldots$

- go in the $(u, v)$ map if the calculations become easier (think of the convolution that becomes a product). 
- go back in the initial map by inverting the expression in $(u, v)$ thanks to the properties.

In this paper, the sequences $a_{n, m}$ will be probability functions and so, ordinary generating functions are just probability generating functions. In this case we have the convergence of the bivariate sums at least if $|u|<1$ and $|v|<1$.

\section{THE MODEL}

We are going to study the random vector $(N, M)$ of counting variables. We will obtain the distribution of $(N, M)$ by mixing the conditional distribution of $(N, M)$ with a random variable $\Lambda$ with distribution function $U(\lambda)$ :

$$
\mathbb{P}(N=n, M=m)=\int_{0}^{\infty} \mathbb{P}(N=n, M=m \mid \Lambda=\lambda) d U(\lambda) .
$$

Furthermore we assume that

- Conditionally on $A$ the random variables $N$ and $M$ are independent.

- The conditional distributions of $N$ and $M$ given that $A=\lambda$ are univariate Poisson with parameter respectively $\lambda$ and $\beta \lambda$.

The probability generating function of $(N, M), \psi_{N, M}(u, v)=\mathbb{E}\left[u^{N} v^{M}\right]$, writes

$$
\psi_{N, M}(u, v)=\int_{0}^{\infty} e^{\lambda(u-1)+\beta \lambda(v-1)} d U(\lambda) .
$$

Kemp (1981) introduced the notion of Homogeneous Bivariate Distribution:

Definition: A bivariate probability generating function $\psi(u, v)$ is said to be of the homogeneous type if

with

$$
\psi(u, v)=H\left(\sigma_{1} u+\sigma_{2} v\right)
$$

$$
H\left(\sigma_{1}+\sigma_{2}\right)=1
$$

If one chooses $H$ such that

$$
H(x)=\int_{0}^{\infty} e^{-\lambda(1+\beta)} e^{\lambda x} d U(\lambda),
$$

we immediately get that $(N, M)$ is a Bivariate Homogeneous Distribution with $\sigma_{1}=1$ and $\sigma_{2}=\beta$.

Kocherlakota and Kocherlakota (1992) have given the following characterization theorem:

Theorem: The probability generating function $\psi_{N, M}(u, v)$ is of the homogeneous type if and only if the conditional distribution of $N$ given $N+M=z$ is Binomial distributed: $B i\left(z, \frac{\sigma_{1}}{\sigma_{1}+\sigma_{2}}\right)$. 
In our case we have that

$$
N \mid N+M=z \sim B i\left(z, \frac{1}{1+\beta}\right),
$$

a result that also has been obtained by Partrat (1994) and Hesselager (1996). From Hesselager (1996) it is possible to extend the result of Kocherlakota and Kocherlakota (1992) by

$$
\begin{aligned}
\rho_{1} & =\frac{\sigma_{1}}{\sigma_{1}+\sigma_{2}}, \\
\rho_{2} & =\frac{\sigma_{2}}{\sigma_{1}+\sigma_{2}}, \\
\psi_{N, M}(u, v) & =\psi_{N+M}\left(\rho_{1} u+\rho_{2} v\right),
\end{aligned}
$$

where $\psi_{N+M}(u)=\sum_{i=0}^{\infty} \mathbb{P}[N+M=i] u^{i}$ is the probability generating function of $N+M$.

\section{The Mixed Bivariate Hofmann Distribution}

Walhin and Paris (2000b) described the Hofmann Distribution. Let us recall some concepts.

Let $N(t)$ be the number of claims occurring in the time interval $(0, t]$ with $N(0)=0$. Assume $N(t)$ is an infinitely divisible Mixed Poisson process (see Grandell (1997)) for which

and

$$
\Pi(n, t)=\mathbb{P}[N(t)=n]=\int_{0}^{\infty} e^{-\lambda t} \frac{(\lambda t)^{n}}{n !} d U(\lambda),
$$

$$
\Pi(0, t)=\mathbb{P}[N(t)=0]=e^{-\theta(t)},
$$

where $\theta(t)$ is a Bernstein function:

$$
\begin{gathered}
\theta(t) \geq 0, \\
\theta(0)=0, \\
\frac{d}{d t} \theta(t) \text { completely monotone. }
\end{gathered}
$$

The probability generating function of $N(t), \psi_{N(t)}(u)=\mathbb{E}\left[u^{N(t)}\right]$, writes

$$
\psi_{N(t)}(u)=\Pi(0, t-t u) .
$$

With the particular choice

$$
\frac{d}{d t} \theta(t)=\frac{p}{(1+c t)^{a}}, \quad p>0, c>0, a \geq 0,
$$


we have a Hofmann process (see Hofmann (1955) or Kestemont and Paris (1985)).

By integration, one has

$$
\begin{aligned}
& \theta(t)=\frac{p}{c(1-a)}\left[(1+c t)^{1-a}-1\right] \text { if } a \neq 1, \\
& \theta(t)=\frac{p}{c} \ln (1+c t) \text { by continuity for } a=1
\end{aligned}
$$

Particular cases of interest are: $a=0$ (Poisson), $a=0.5$ (Poisson Inverse Gaussian), $a=1$ (Negative Binomial), $a=2$ (Polya-Aeppli), $a \rightarrow \infty, c \rightarrow 0, a c \rightarrow b$ (Neymann Type A).

For fixed $t$, it is possible to express the random variable $N(t)$ in the form of a compound Poisson distribution:

$$
N(t)=\sum_{i=1}^{L(t)} \Xi_{i}
$$

where $L(t)$ is Poisson distributed with mean $\theta(t)$, independent of the $\Xi_{i}$ which are independent and identically distributed. Moreover as

$$
\frac{\mathbb{P}[\Xi=\xi]}{\mathbb{P}[\Xi=\xi-1]}=r+\frac{s}{\xi}, \quad \xi>1,
$$

the probability distribution of $\Xi$ is a member of the $(r, s, 1)$ class of counting distributions. The $(r, s, 1)$ class is just a reparametrization of the classical $(a, b, 1)$ class in order to avoid confusion with the $a$ of the Hofmann Distribution.

Thanks to this property, it is possible to use the Panjer algorithm in order to evaluate the aggregate distribution of $S$ :

$$
S=\sum_{i=1}^{N(t)} X_{i}
$$

It is however necessary to apply the algorithm two times: we first introduce the random variable

$$
U=\sum_{i=1}^{\Xi} X_{i}
$$

and then

$$
S=\sum_{i=1}^{L(t)} U_{i}
$$

The distribution of $U$ can be evaluated by the extended Panjer algorithm (see Sundt and Jewell (1981)) whereas the distribution of $S$ can be evaluated by the Panjer algorithm (see Panjer (1981)).

For fixed $t$, in the sequel we will write $\Pi_{p, c, a}(n, t)$ for $\Pi(n, t)$ in order to specify the use of the Hofmann Distribution $H_{o}(p, c, a)$. 
From now on let us fix $t=1$ and let us use the Hofmann Distribution in our bivariate case. Let us assume that $A$ is the mixing variable leading to the Hofmann Distribution.

From (1) and (2) we immediately get

$$
\mathbb{P}(N=n, M=m)=\frac{(n+m) !}{n ! m !} \frac{\beta^{m}}{(1+\beta)^{n+m}} \Pi(n+m, 1+\beta),
$$

where it is easy to see that

$$
\Pi_{p, c, a}(n+m, 1+\beta)=\Pi_{(1+\beta) p,(1+\beta) c, a}(n+m, 1) .
$$

In fact our model introduces dependency such that:

$$
\begin{aligned}
N & \sim H o(p, c, a), \\
M & \sim H o(p \beta, c \beta, a), \\
N+M & \sim H o(p(1+\beta), c(1+\beta), a) .
\end{aligned}
$$

This clearly generalizes the reasoning of Partrat (1994) where only $A$ Gamma or Inverse Gaussian distributed are considered.

\section{ESTIMATION OF THE PARAMETERS}

Let us assume that we have observed a sample $\left(n_{i}, m_{i}\right), 1 \leq i \leq q$ of $(N, M)$. The log-likelihood writes

$$
\begin{aligned}
l(\beta, p, c, a) & =\ln \prod_{i=1}^{q} \mathbb{P}\left(N=n_{i}, M=m_{i}\right) \\
& =C+\ln \beta \sum_{i=1}^{q} m_{i}-\ln (1+\beta) \sum_{i=1}^{q}\left(n_{i}+m_{i}\right)+l_{n+m}(\beta, p, c, a),
\end{aligned}
$$

where $l_{n+m}(\beta, p, c, a)$ is the log-likelihood for the univariate Hofmann Distribution $H o((1+\beta) p,(1+\beta) c, a)$ with the sample $\left(n_{i}+m_{i}\right), 1 \leq i \leq q$ and $C$ is a term that does not depend on the unknown parameters.

As shown in Besson and Partrat (1992), $\frac{\partial}{\partial \beta} l_{n+m}(\beta, p, c, a)=0$ at the maximum likelihood estimate. So we immediately get

$$
\hat{\beta}=\frac{\bar{m}}{\bar{n}},
$$

where $\bar{n}$ (resp. $\bar{m}$ ) is the experimental mean of $N$ (resp. $M$ ).

By standard results on the univariate Hofmann Distribution (see Hürlimann (1990)), we know that the maximum likelihood estimate of the mean is the observed frequency. Therefore maximizing $l_{n+m}(\beta, p, c, a)$ implies that

$$
\hat{p}(1+\hat{\beta})=\bar{n}+\bar{m} \text {. }
$$


So the estimates $\hat{p}$ and $\hat{\beta}$ are derived analytically. The other two estimates $\hat{c}$ and $\hat{a}$ have to be found by standard numerical maximization techniques. Note that one has to be careful because the likelihood may be very flat and local extrema are not excluded.

\section{A STABlE TWO-STAGE RECURSION FOR THE AGGREGATE CLAIMS DISTRIBUTION}

In the conclusion of his paper, Partrat (1994) addresses the problem of finding recursions like Panjer's recursion (see Panjer (1981)) in order to obtain the distribution of the aggregate claims.

Let $X_{i}$ be the random variable representing the $i$ th claim amount of type $N$ and $Y_{i}$ the random variable representing the $i$ th claim amount of type $M$. We will assume, as usual, that the $X_{i}$ and $Y_{i}$ are mutually independent random variables. They are also arithmetic. The $X_{i}$ are identically distributed. The $Y_{i}$ are identically distributed. We also assume that the $X_{i}$ and $Y_{i}$ are independent of $N$ and $M$. We are interested in the distribution of

$$
(S, T)=\left(\sum_{i=1}^{N} X_{i}, \sum_{i=1}^{M} Y_{i}\right)
$$

In the case of the Mixed Bivariate Negative Binomial Distribution the answer to Partrat's question was given by Hesselager (1996). In his paper, Hesselager (1996) gives a stable algorithm for the evaluation of the joint probability function of $(S, T)$ for the particular case of the Mixed Bivariate Negative Binomial Distribution, i.e. when $A$ is Gamma distributed.

In this section we will use the same methodology as in Hesselager (1996) in order to derive stable algorithms for the distribution of $(S, T)$. As we know that $U$ is infinitely divisible, it follows from Maceda (1948) that the distribution of $(N, M)$ is also infinitely divisible.

Then we know from Sundt $(1999 \mathrm{a})$ that $(N, M)$ can be interpreted as a bivariate Compound Poisson distribution:

$$
(N, M)=\left(\sum_{i=1}^{L} \Xi_{i}, \sum_{i=1}^{L} \Omega_{i}\right)
$$

where the $\Xi_{i}$ and $\Omega_{i}$ are not independent and $L$ is Poisson distributed independently of the $\left(\Xi_{i}, \Omega_{i}\right)$.

To be able to use the bivariate Panjer algorithm, we introduce the auxiliary vectors

and then

$$
(U, V)=\left(\sum_{i=1}^{\Xi} X_{i}, \sum_{i=1}^{\Omega} Y_{i}\right)
$$

$$
(S, T)=\left(\sum_{i=1}^{L} U_{i}, \sum_{i=1}^{L} V_{i}\right)
$$


We will use the following notation:

\section{Probability functions}

$$
\begin{aligned}
\mathbb{P}(N=n, M=m) & =p(n, m), \\
\mathbb{P}(K=k) & =f_{K}(k),(K=N+M), \\
\mathbb{P}(X=x) & =f_{X}(x), \\
\mathbb{P}(Y=y) & =f_{Y}(y), \\
\mathbb{P}(S=s, T=t) & =f_{S, T}(s, t), \\
\mathbb{P}(\Xi=n, \Omega=m) & =f_{\Xi, \Omega}(n, m) .
\end{aligned}
$$

Probability generating functions

$$
\begin{aligned}
\psi_{X}(u) & =\sum_{x=0}^{\infty} f_{X}(x) u^{x}, \\
\psi_{Y}(v) & =\sum_{y=0}^{\infty} f_{Y}(y) v^{y}, \\
\psi_{K}(u) & =\sum_{r=0}^{\infty} f_{K}(k) u^{k}, \\
\psi_{N, M}(u, v) & =\sum_{n=0}^{\infty} \sum_{m=0}^{\infty} p(n, m) u^{n} v^{m}, \\
\psi_{S, T}(u, v) & =\sum_{x=0}^{\infty} \sum_{y=0}^{\infty} f_{S, T}(x, y) u^{x} v^{y}, \\
\psi_{\Xi, \Omega}(u, v) & =\sum_{n=0}^{\infty} \sum_{m=0}^{\infty} f_{\Xi, \Omega}(n, m) u^{n} v^{m} .
\end{aligned}
$$

The bivariate Panjer's algorithm described in Walhin and Paris (2000a) as well as in Sundt (1999b) and Ambagaspitya (1999) will be used in order to find the distribution of $(S, T)$ knowing the distribution of $(U, V)$.

In a first time we are interested in deriving the distribution of $(U, V)$. Therefore we first need to derive the distribution of $(\Xi, \Omega)$.

Remember that we have

$$
\psi_{N, M}(u, v)=\psi_{N+M}\left(\rho_{1} u+\rho_{2} v\right) .
$$

We find

$$
\begin{aligned}
\psi_{N, M}(u, v) & =e^{-\theta\left(1+\beta-(1+\beta)\left(\rho_{1} u+\rho_{2} v\right)\right)}, \\
& =e^{-\theta(1+\beta)\left(1-\psi_{\Xi, \Omega}(u, v)\right)},
\end{aligned}
$$

where

$$
\begin{aligned}
& \psi_{\Xi, \Omega}(0,0)=0, \\
& \psi_{\Xi, \Omega}(u, v)=1-\frac{\theta\left((1+\beta)\left(1-\left(\rho_{1} u+\rho_{2} v\right)\right)\right)}{\theta(1+\beta)} .
\end{aligned}
$$


$(\Xi, \Omega)$ also has a bivariate homogeneous distribution. Indeed

$$
\begin{aligned}
\psi_{\Xi, \Omega}(u, v) & =G\left(\rho_{1} u+\rho_{2} v\right), \\
G(x) & =1-\frac{\theta((1+\beta)(1-x))}{\theta(1+\beta)}, \\
G\left(\rho_{1}+\rho_{2}\right) & =1 .
\end{aligned}
$$

The Taylor expansion around $(1+\beta)$ of $(5)$ gives after a few calculations

$$
f_{\Xi, \Omega}(n, m)=\frac{\theta^{(m+n)}(1+\beta)}{\theta(1+\beta)} \frac{(-1)^{m+n-1}}{(m+n) !} \beta^{m} \frac{(n+m) !}{n ! m !} .
$$

As we have

$$
\begin{aligned}
\theta(1+\beta) & =\frac{p}{c(1-\alpha)}\left[(1+c(1+\beta))^{1-a}-1\right], \\
\theta^{(n+m)}(1+\beta) & =(-1)^{n+m-1} p c^{n+m-1} \frac{\Gamma(\alpha+m+n-1)}{\Gamma(\alpha)}(1+c(1+\beta))^{1-a-n-m},
\end{aligned}
$$

we immediately get

$$
\begin{aligned}
f_{\Xi, \Omega}(n, m) & =\frac{(n+m) !}{n ! m !} \frac{c^{n+m}}{(n+m) !} \frac{1-a}{\Gamma(a)} \beta^{m} \Gamma(a+m+n-1) \frac{[1+c(1+\beta)]^{1-a-n-m}}{[1+c(1+\beta)]^{1-a}-1} \\
& =\frac{(n+m) !}{n ! m !} \rho_{1}^{n} \rho_{2}^{m} \mathbb{P}(W=n+m),
\end{aligned}
$$

with

$$
W=\Xi+\Omega \text {, }
$$

and

$$
\begin{aligned}
& \rho_{1}=\frac{1}{1+\beta}, \\
& \rho_{2}=\frac{\beta}{1+\beta} .
\end{aligned}
$$

Indeed

$$
\begin{aligned}
\psi_{W}(u) & =\psi_{\Xi, \Omega}(u, u) \\
& =1-\frac{\theta((1+\beta)(1-u))}{\theta(1+\beta)}
\end{aligned}
$$

A Taylor expansion around $(1+\beta)$ of (6) immediately shows that

$$
\mathbb{P}(W=w)=\frac{c^{w}}{w !} \frac{1-\alpha}{\Gamma(\alpha)} \Gamma(\alpha+w-1)(1+\beta)^{w} \frac{[1+c(1+\beta)]^{1-a-w}}{[1+c(1+\beta)]^{1-a}-1} .
$$


With the particular form of the distribution of $W$, we immediately find, with $f_{W}(w)=\mathbb{P}(W=w)$,

$$
\frac{f_{W}(w)}{f_{W}(w-1)}=\frac{c(1+\beta)}{1+c(1+\beta)}+\frac{c(1+\beta)(\alpha-2)}{1+c(1+\beta)} \frac{1}{w}, w>1,
$$

which is well in the form of the $(r, s, 1)$ class with

$$
\begin{aligned}
r & =\frac{c(1+\beta)}{1+c(1+\beta)}, \\
s & =\frac{c(1+\beta)(a-2)}{1+c(1+\beta)}, \\
f_{W}(0) & =0, \\
f_{W}(1) & =c(1+\beta)(1-a) \frac{[1+c(1+\beta)]^{-a}}{[1+c(1+\beta)]^{1-a}-1} .
\end{aligned}
$$

Now let us study the distribution of

$$
(U, V)=\left(X_{1}+\ldots+X_{\Xi}, Y_{1}+\ldots+Y_{\Omega}\right) .
$$

As $W$ is a member of the $(r, s, 1)$ class we have:

$$
[1-r u] \frac{d}{d u} \psi_{W}(u)=f_{W}(1)+(r+s) \psi_{W}(u) .
$$

We are now able to extend Hesselager's methodology to find the aggregate claims distribution.

\section{Theorem 1}

We have

$$
\begin{aligned}
f_{\Xi, \Omega}(1,0)= & \rho_{1} f_{W}(1), \\
f_{\Xi, \Omega}(n, m)= & \rho_{1}\left(r+\frac{s}{n}\right) f_{\Xi, \Omega}(n-1, m) \\
& +r \rho_{2} f_{\Xi, \Omega}(n, m-1), n \geq 1 \text { unless if }(n, m)=(1,0), \\
f_{\Xi, \Omega}(0,1)= & \rho_{2} f_{W}(1), \\
f_{\Xi, \Omega}(n, m)= & \rho_{2}\left(r+\frac{s}{n}\right) f_{\Xi, \Omega}(n, m-1) \\
& +r \rho_{1} f_{\Xi, \Omega}(n-1, m), m \geq 1 \text { unless if }(n, m)=(0,1),
\end{aligned}
$$

Proof

We already noticed that

$$
\psi_{\Xi, \Omega}(u, v)=\psi_{W}\left(\rho_{1} u+\rho_{2} v\right)
$$


By differentiating (12) with respect to $u$, and using (7), we get

$$
\left(1-r \rho_{1} u-r \rho_{2} v\right) \frac{\partial}{\partial u} \psi_{\Xi, \Omega}(u, v)=\rho_{1}\left(f_{W}(1)+(r+s) \psi_{\Xi, \Omega}(u, v)\right) .
$$

Inverting this expression we immediately get (8) and (9).

(10) and (11) are derived similarly.

\section{Theorem 2}

We have

$$
\begin{aligned}
f_{U, V}(0,0)= & 1-\frac{\theta\left((1+\beta)\left(1-\left(\rho_{1} f_{X}(0)+\rho_{2} f_{Y}(0)\right)\right)\right)}{\theta(1+\beta)} \\
f_{U, V}(x, 0)= & \frac{1}{1-r \rho_{1} f_{X}(0)}\left(\rho_{1} f_{W}(1) f_{X}(x)\right. \\
& \left.+\rho_{1} \sum_{i=1}^{x}\left(r+\frac{s i}{x}\right) f_{X}(i) f_{U, V}(x-i, 0)\right), x>0, \\
f_{U, V}(0, y)= & \frac{1}{1-r \rho_{2} f_{Y}(0)}\left(\rho_{2} f_{W}(1) f_{Y}(y)\right. \\
& \left.+\rho_{2} \sum_{i=1}^{y}\left(r+\frac{s i}{y}\right) f_{Y}(i) f_{U, V}(0, y-i)\right), y>0, \\
& \left.+\rho_{2} r \sum_{j=1}^{y} f_{Y}(j) f_{U, V}(x, y-j)\right), x>0, y>0, \\
f_{U, V}(x, y)= & \frac{1}{1-r \rho_{1} f_{X}(0)-r \rho_{2} f_{Y}(0)}\left(\rho_{1} \sum_{i=1}^{x}\left(r+\frac{s i}{x}\right) f_{X}(i) f_{U, V}(x-i, y)\right. \\
& \left.+\rho_{1} r \sum_{j=1}^{x} f_{X}(j) f_{U, V}(x-j, y)\right), y>0, x>0 .
\end{aligned}
$$

\section{Proof}

As $f_{U, V}(0,0)=\psi_{\Xi, \Omega}\left(f_{X}(0), f_{Y}(0)\right)$ we immediately find (13) by using equation (5). From (9) we get

$$
\begin{aligned}
f_{\Xi, \Omega}(n, m)= & \rho_{1}\left(r+\frac{s}{n}\right) f_{\Xi, \Omega}(n-1, m)+r \rho_{2} f_{\Xi, \Omega}(n, m-1) \\
\Leftrightarrow & \\
n f_{\Xi, \Omega}(n, m)= & r \rho_{1}(n-1) f_{\Xi, \Omega}(n-1, m) \\
& +\rho_{1}(r+s) f_{\Xi, \Omega}(n-1, m)+r \rho_{2} n f_{\Xi, \Omega}(n, m-1) .
\end{aligned}
$$


Multiplying both sides of (19) by $u \psi_{X}^{n-1}(u) \frac{d}{d u} \psi_{X}(u) \psi_{Y}^{m}(v)$ and summing on $n=$ $1 \rightarrow \infty, m=1 \rightarrow \infty$ gives

$$
\begin{aligned}
u \frac{\partial}{\partial u} \psi_{U, V}(u, v)= & r \rho_{1} u \frac{\partial}{\partial u} \psi_{U, V}(u, v) \psi_{X}(u) \\
& +(r+s) \rho_{1} u \frac{d}{d u} \psi_{X}(u) \psi_{U, V}(u, v)+r \rho_{2} \psi_{U, V}(u, v) \psi_{Y}(v)
\end{aligned}
$$

Inverting and rearranging this expression gives (16).

Multiplying both sides of (19) by $u \psi_{X}^{n-1}(u) \frac{d}{d u} \psi_{X}(u) \psi_{Y}^{m}(0)$, summing on $n=2$ $\rightarrow \infty, m=0 \rightarrow \infty$ and adding $f_{\Xi, \Omega}(1,0) u \frac{d}{d u} \psi_{X}(u)$ on both sides gives

$$
\begin{aligned}
u \frac{\partial}{\partial u} \psi_{U, V}(u, v)= & \rho_{1} f_{W}(1) \psi_{X}(u)+r \rho_{1} u \frac{\partial}{\partial u} \psi_{U, V}(u, v) \psi_{X}(u) \\
& +(r+s) \rho_{1} u \frac{d}{d u} \psi_{X}(u) \psi_{U, V}(u, v) .
\end{aligned}
$$

Inverting and rearranging this expression gives (14).

(15) and (17) are derived similarly.

Knowing the distribution of $(U, V)$ it remains to evaluate the distribution of

$$
(S, T)=\left(\sum_{i=1}^{L} U_{i}, \sum_{i=1}^{L} V_{i}\right)
$$

This is easily done with the bivariate Panjer's algorithm.

We have

$$
\begin{aligned}
& f_{S, T}(0,0)=e^{-\theta(1+\beta)\left(1-f_{U, V}(0,0)\right)}, \\
& f_{S, T}(s, t)=\sum_{x}^{s} \sum_{y}^{t}\left[\theta(1+\beta) \frac{x}{s}\right] f_{S, T}(s-x, t-y) f_{U, V}(x, y), s \geq 1, \\
& f_{S, T}(s, t)=\sum_{x}^{s} \sum_{y}^{t}\left[\theta(1+\beta) \frac{y}{t}\right] f_{S, T}(s-x, t-y) f_{U, V}(x, y), t \geq 1,
\end{aligned}
$$

where we use the following notation:

$$
\sum_{x, y}^{s, t} g(x, y)=\sum_{x=0}^{s} \sum_{y=0}^{t} g(x, y)-g(0,0)
$$




\section{NUMERICAL APPLICATIONS}

In this section we fit two data sets given in Partrat (1994).

Data set 1 gives the yearly frequencies of hurricanes affecting two zones (zone 1 and zone 3) of the United States.

Data set 2 gives the yearly frequencies of an automobile third party liability portfolio, divided in material damage (type 1) and bodily injury (type 2 ) claims.

TABLE 1

DATA SET 1

\begin{tabular}{llllll}
\hline \hline & & zone3 & & & \\
\hline zone1 & & 0 & 1 & 2 & 3 \\
\hline 0 & $o b s$ & 27 & 9 & 3 & 2 \\
& $a=0$ & 27.67 & 13.04 & 3.08 & 0.49 \\
& $a=0.0057$ & 27.59 & 13.05 & 3.09 & 0.49 \\
\hline 1 & $o b s$ & 24 & 13 & 1 & 0 \\
& $a=0$ & 20.45 & 9.66 & 2.29 & 0.36 \\
& $a=0.0057$ & 20.47 & 9.69 & 2.29 & 0.36 \\
\hline 2 & $o b s$ & 8 & 2 & 1 & 0 \\
& $a=0$ & 7.57 & 3.59 & 0.85 & 0.14 \\
& $a=0.0057$ & 7.59 & 3.59 & 0.85 & 0.13 \\
\hline 3 & $o b s$ & 1 & 0 & 2 & 0 \\
& $a=0$ & 1.88 & 0.89 & 0.21 & 0.03 \\
& $a=0.0057$ & 1.88 & 0.89 & 0.21 & 0.03 \\
\hline \hline
\end{tabular}

For this data set, the maximum likelihood procedure for the Mixed Bivariate Hofmann Distribution $(a=0.0057)$ gives almost the Bivariate Independent Poisson Distribution $(\alpha=0)$.

The characteristics of the fit are given in the next table. In order to compute the $\chi^{2}$ statistic, some cells have been grouped in order that the theoretical frequencies are all larger than 1 and about $80 \%$ of the theoretical frequencies are larger than 5 .

In the present case we work with 8 classes: $(0,0),(0,1),(1,0),(1,1),(2,0)$, $(0,2+)$ and $(1,2+),(2,1+),(3+, 0+)$.

TABLE 2

Parameter estimates, Loglikelihood and $\chi^{2}$ Test - Data Set 1

\begin{tabular}{lll}
\hline \hline & BIPD & MBHD \\
\hline$\beta$ & 0.6377 & 0.6377 \\
$p$ & 0.7419 & 0.7419 \\
$c$ & & 0.5137 \\
$\alpha$ & 0 & 0.0058 \\
\hline$\lambda$ & -187.9615 & -187.9607 \\
$\chi^{2}$ & 3.73 & 3.77 \\
$d f$ & 5 & 3 \\
p-value & 0.589 & 0.287 \\
\hline \hline
\end{tabular}


A likelihood ratio test does not reject the null hypothesis that Bivariate Poisson Distribution is adequate against the more general model Mixed Bivariate Hofmann Distribution. Within the latter model we cannot reject the fact that both random variables are independent. In this case the principle of parsimony indicates that the Bivariate Independent Poisson Distribution (BIPD) should be retained. Now let us study our second data set:

TABLE 3

DATA SET 2

\begin{tabular}{|c|c|c|c|c|}
\hline \multirow[b]{2}{*}{ Material damage } & \multicolumn{4}{|c|}{ Bodily injury } \\
\hline & & 0 & 1 & 2 \\
\hline$\overline{0}$ & $\begin{array}{l}\text { obs } \\
a=0 \\
a=1 \\
a=0.5 \\
a=0.2982\end{array}$ & $\begin{array}{l}171345 \\
171086.9 \\
171348.8 \\
171348.7 \\
171345.8\end{array}$ & $\begin{array}{l}918 \\
946.0 \\
897.1 \\
897.5 \\
898.6\end{array}$ & $\begin{array}{l}2 \\
2.6 \\
4.7 \\
4.6 \\
4.5\end{array}$ \\
\hline$\overline{1}$ & $\begin{array}{l}\text { obs } \\
a=0 \\
a=1 \\
a=0.5 \\
a=0.2982\end{array}$ & $\begin{array}{l}8273 \\
8726.4 \\
8275.5 \\
8279.5 \\
8289.4 \\
\end{array}$ & $\begin{array}{l}73 \\
48.2 \\
86.3 \\
84.9 \\
82.8\end{array}$ & $\begin{array}{l}0 \\
0.1 \\
0.7 \\
0.8 \\
0.8\end{array}$ \\
\hline 2 & $\begin{array}{l}\text { obs } \\
a=0 \\
a=1 \\
a=0.5 \\
a=0.2982\end{array}$ & $\begin{array}{l}389 \\
222.5 \\
398.2 \\
391.5 \\
381.9 \\
\end{array}$ & $\begin{array}{l}5 \\
1.2 \\
6.2 \\
6.9 \\
7.6 \\
\end{array}$ & $\begin{array}{l}0 \\
0.0 \\
0.1 \\
0.1 \\
0.1 \\
\end{array}$ \\
\hline 3 & $\begin{array}{l}o b s \\
a=0 \\
a=1 \\
a=0.5 \\
a=0.2982\end{array}$ & $\begin{array}{l}31 \\
3.8 \\
19.1 \\
21.3 \\
23.5\end{array}$ & $\begin{array}{l}1 \\
0.0 \\
0.4 \\
0.6 \\
0.8\end{array}$ & $\begin{array}{l}0 \\
0.0 \\
0 \\
0 \\
0\end{array}$ \\
\hline$\overline{4}$ & $\begin{array}{l}\text { obs } \\
a=0 \\
a=1 \\
a=0.5 \\
a=0.2982\end{array}$ & $\begin{array}{l}1 \\
0.0 \\
0.9 \\
1.3 \\
1.9\end{array}$ & $\begin{array}{l}0 \\
0.0 \\
0.0 \\
0.0 \\
0.1\end{array}$ & $\begin{array}{l}0 \\
0.0 \\
0.0 \\
0.0 \\
0.0\end{array}$ \\
\hline
\end{tabular}

TABLE 4

Parameter estimates, loglikelihood and $\chi^{2}$ Test - Data SET 2

\begin{tabular}{lllll}
\hline \hline & MBPD & MBNBD & MBPIGD & MBHD \\
\hline$\beta$ & 0.1084 & 0.1084 & 0.1084 & 0.1084 \\
$p$ & 0.0510 & 0.0510 & 0.0510 & 0.0510 \\
$c$ & & 0.8934 & 1.8235 & 3.0695 \\
$a$ & 0 & 1 & 0.5 & 0.3006 \\
\hline$\lambda$ & 43251.57 & -43143.09 & 43141.79 & -41141.27 \\
$\chi^{2}$ & 369.76 & 11.54 & 8.72 & 7.44 \\
$d f$ & 5 & 4 & 4 & 3 \\
p-value & 0 & 0.021 & 0.068 & 0.059 \\
\hline \hline
\end{tabular}


The MBPD and MBNBD are rejected at the 5\% level. The MBPIGB and MBHD are not rejected at the $5 \%$ level. The grouped cells are: $(0,0),(0,1)$, $(1,0),(1,1),(2,0),(2,1),(3,0)$, the rest.

Let us work with the portfolio given in table 3 and the Mixed Bivariate Hofmann fit of this portfolio.

Let us assume that the distributions $X$ (material damage) and $Y$ (bodily injury) are given by:

TABLE 5

Claims Distributions

\begin{tabular}{llll}
\hline \hline $\boldsymbol{X}$ & $\boldsymbol{f}_{\boldsymbol{X}}(\boldsymbol{x})$ & $\boldsymbol{Y}$ & $\boldsymbol{f}_{\boldsymbol{Y}}(\boldsymbol{y})$ \\
\hline 1 & 0.2 & 5 & 0.2 \\
2 & 0.2 & 10 & 0.36 \\
3 & 0.2 & 20 & 0.22 \\
4 & 0.1 & 50 & 0.11 \\
5 & 0.1 & 100 & 0.11 \\
10 & 0.1 & & \\
20 & 0.1 & & \\
\hline \hline
\end{tabular}

We find the aggregate claims distribution:

TABLE 6

AgGregate Claims distribution

\begin{tabular}{lllll}
\hline & $S=0$ & $S=1$ & $S=2$ & $S=3$ \\
\hline$T=0$ & 0.9410275 & 0.0010876 & 0.0019589 & 0.0000042 \\
$T=5$ & 0.0100336 & 0.0000219 & 0.0000395 & 0.0000001 \\
$T=10$ & 0.0101349 & 0.0000223 & 0.0000403 & 0.0000001 \\
$T=15$ & 0.0102375 & 0.0000228 & 0.0000411 & 0.0000001 \\
$\ldots$ & & & & \\
\hline \hline
\end{tabular}

Obviously, as the span of the $Y$ claims is 5, it is most convenient (and less time consuming) to rescale the $Y$ claims by division by 5 and then to revert the scaling for the final bivariate aggregate claims distribution.

\section{CONCLUSION}

In this paper we have extended the use of traditional Mixed Bivariate Independent Poisson Distributions into a general family of bivariate counting distributions. This family has interesting properties. On the one hand it authorizes a maximum likelihood estimation in a univariate setting. On the other hand it gives stable algorithms for the evaluation of the bivariate aggregate claims distribution.

The fits of some insurance portfolios are improved thanks to the use of the Mixed Bivariate Hofmann Distribution. 


\section{REMARK}

A first version of this paper has been presented at the Royal Statistical Society Conference 1999, Warwick.

Three anonymous referees are greatly acknowledged for very detailed and constructive comments on earlier versions of this paper.

\section{REFERENCES}

Ambagaspitya, R.S. (1999) On the Distributions of Two Classes of Correlated Aggregate Claims. Insurance: Mathematics and Economics, 24, 301-308.

Besson, J.L. and Partrat, C. (1992) Trend et Systèmes de Bonus-Malus. Astin Bulletin, 22, 11-31.

Grandell, J. (1997) Mixed Poisson Processes. Chapman and Hall.

Hesselager, O. (1996) Recursions for Certain Bivariate Counting Distributions and their Compound Distributions. Astin Bulletin, 26, 35-52.

Hofmann, M. (1955) Uber zusammengesetzte Poisson-Prozesse und ihre Anwendungen in der Unfallversicherung. Bulletin of the Swiss Actuaries, 55, 499-575.

HürlimanN, W. (1990) On Maximum Likelihood Estimation for Count Data Models. Insurance: Mathematics and Economics, 9, 39-49.

KemP, A.W. (1981) Computer Sampling from Homogeneous Bivariate Discrete Distributions. ASA Proceedings of the Statistical Computing Section, pages 173-175.

Kestemont, R.M. and Paris J. (1985) Sur l'Ajustement du Nombre de Sinistres. Bulletin of the Swiss Actuaries, 85, 157-164.

Kocherlakota, S. and Kocherlakota, K. (1992) Bivariate Discrete Distributions. Marcel Dekker, New-York.

MACEDA, E. (1948) On the Compound and Generalized Poisson Distributions. Annals of Mathematical Statistics, 19, 414-416.

PANJer, H.H. (1981) Recursive Evaluation of a Family of Compound Distributions. Astin Bulletin, 12, 22-26.

PANJer H.H. and Willmot, G.E. (1992) Insurance Risk Models. Society of Actuaries.

PARTRAT, C. (1994) Compound Model for Two Dependent Kinds of Claims. Insurance: Mathematics and Economics, 15, 219-231.

Sund, B. (1999a) Multivariate Compound Poisson Distributions and Infinite Divisibility. Statistical report 33, Department of Mathematics, University of Bergen.

Sundt, B. (1999b) On Multivariate Panjer Recursions. Astin Bulletin, 29, 29-45.

Sund, B. and JEwell, W.S. (1981) Further Results on Recursive Evaluation of Compound Distributions. Astin Bulletin, 12, 27-39.

Walhin, J.F. and PARIS, J. (2000a) The Effect of Excess of Loss Reinsurance with Reinstatements on the Cedent's Portfolio. Blatter Deutsche Gesellschaft für Versicherungsmathematik, 24, 615-627.

WALhin, J.F. and PARIS, J. (2000b) A Large Family of Discrete and Overdispersed Probability Laws. submitted.

Walhin, J.F. and Paris, J. (2000c) Recursive Formulae for Some Bivariate Counting Distributions Obtained by the Trivariate Reduction Method. Astin Bulletin, 30, 141-155.

JEAN-FrançOIS WALHIN

SECURA Belgian Re

Rue Montoyer 12

1000 Bruxelles

E-mail: jfw@secura-re.com 